\title{
Meningeal carcinomatosis and blindness
}

\author{
PAUL H. ALTROCCHI AND PAUL B. ECKMAN \\ From the Section of Neurology, Palo Alto Medical Clinic, \\ Palo Alto, California 94301, U.S.A.
}

SUMMARY The clinical syndrome of meningeal carcinomatosis includes headache, dementia, $\stackrel{\mathbb{D}}{\stackrel{0}{\varrho}}$

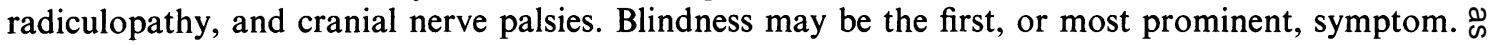
When blindness occurs in adult life, meningeal carcinomatosis should be included in the differential $\vec{\circ}$ diagnosis, even in the absence of other symptoms and in the absence of known malignancy. Although all pathophysiological mechanisms of the blindness in meningeal carcinomatosis have not yet been $\vec{\omega}$ elucidated, optic nerve involvement by meningeal tumour-cuffing, by chronic papilloedema, and by direct tumour infiltration represent the likeliest causes. In the neuropathological analysis of such cases, the importance of analysing the intra-orbital portion of the optic nerves, in addition to the $\dot{\omega}_{\infty}$ portions of the optic nerve and chiasm usually examined at routine necropsy, is emphasized. A case $i$ is described to illustrate this point, with the only pathological abnormality in the optic nerves being $\stackrel{\sim}{\circ}$ found within $6 \mathrm{~mm}$ of the retina.

The clinical syndrome of meningeal carcinomatosis includes headache, meningeal signs, cranial nerve palsies, dementia, and spinal root signs in varying combinations (Fischer-Williams, Bosanquet, and Daniel, 1955; Grain and Karr, 1955). Visual failure as an important component of the syndrome generally received insufficient emphasis in the ophthalmological and neurological literature (Dixon, Kerr, and Sharp, 1946; Grain and Karr, 1955) until 1955, when FischerWilliams et al. pointed out that partial or complete blindness occurs in one-third of such patients. In 1961, Katz, Valsamis, and Jampel in a review of the neuro-ophthalmological findings in meningeal carcinomatosis described decreased vision in $44 \%$ of cases.

Neuropathological analysis of cases of meningeal carcinomatosis with blindness usually shows meningeal tumour-cuffing around the optic nerves (Fischer-Williams et al., 1955; Heathfield and Williams, 1956; Hawkins and Brown, 1963; Hughes, Adams, and Ilbert, 1963; Kim and Resnick, 1965). Direct infiltration of the optic nerves by tumour cells has only rarely been reported (Kim and Resnick, 1965). In analysing causes for blindness in those cases in whom tumour-cuffing of the optic nerves was minimal,
Fischer-Williams et al., stated: 'It is possibete that there was severe invasion of the optic nerve within the orbit, where they were not examine but this seems unlikely.' Only rarely have the intra-orbital portions of the optic nerves been analysed in such cases. The present case represents the third report in the literature (Terry and Dunphy, 1933; Danis and Brihaye-van Geertruyden, 1952) in which the retinae and adjacent $\overrightarrow{\vec{A}}$ intra-orbital parts of the optic nerves were $\frac{0}{3}$ analysed in a case of meningeal carcinomatosis with blindness, in addition to those parts of the optic nerves and chiasm usually studied neuropathologically. Of special interest is the fact that no tumour-cuffing of the optic nerves was found, and tumour infiltration was found only in the intra-orbital portion of the optic nerves. Unless the optic nerves are studied in their entirety, therefore, from the retinae to the chiasm, direct infiltration by tumour cannot be excluded.

\section{CASE REPORT}

A 59 year old woman (PAMC no. 04-68-17) was referred to the Eye Department of the Palo Alto Medical Clinic in February 1965 because of suddenco flashing lights and floating shadows in the left eye. 
Visual fields and acuity were normal, but the left eye showed blood debris in the vitreous humor, and an extensive area of lattice degeneration in the upper temporal quadrant of the retina, including two retinal holes and a retinal tear. Photocoagulation treatment was carried out with immediate disappearance of the flashing lights and gradual disappearance of the 'floaters'.

In June 1967 her fundi showed changes of early bilateral macular degeneration, with visual acuity in the right eye being $20 / 20$ and the left eye being 20/30. In February 1968 a very anaplastic endometrioid sarcoma was diagnosed by biopsy of a vaginal metastasis. Other metastases were palpable in the pelvis. She was given $4,000 r$ of $x$-ray therapy to the pelvis and lower abdomen, followed six weeks later by complete abdominal hysterectomy and salpingooophorectomy. Because tumour extended to the margins of the incision, she was then treated with intravaginal radium.

In July 1968 she first noted decrease in visual acuity at night. In August 1968, she noted decrease in diurnal visual acuity in the left eye, followed in a few days by slowly progressive blindness in each eye. She also described 'myriads of gold dancing lights in front of my eyes all the time'. By September 1968, her visual acuity was $20 / 40$, O.D., and $20 / 200$, O.S. Radiographs of the skull and optic foramina were normal, as were an intravenous retinal fluorescein angiogram and brain scan. General physical and neurological examinations were normal except for her decreased visual acuity and bilateral paracentral scotomata. The optic discs were normal; her retinal arterioles were very narrow. The pupils were $5 \mathrm{~mm}$ in diameter and reacted well to light and accommodation. The temporal arteries were normal to palpation and her erythrocyte sedimentation rate was normal. Because of her history of previous retinal disease, her night blindness, her lack of concurrent neurological symptoms, and the normal results of her neurological examination, further neurological investigation was deferred.

By December 1968 her corrected visual acuity in the right eye was $20 / 70$, and she could see only gross hand movements on the left. She also had begun to complain of bilateral hip pain. At this point a lumbar puncture was done which showed an opening pressure of $120 \mathrm{~mm}$ of water. The cerebrospinal fluid (CSF) contained 17 lymphocytes, 3 polymorphonuclear leucocytes per cu. $\mathrm{mm}$, a protein of $21 \mathrm{mg} /$ $100 \mathrm{ml}$., a somewhat low sugar of $49 \mathrm{mg} / 100 \mathrm{ml}$. (with a simultaneous blood sugar of $108 \mathrm{mg} / 100 \mathrm{ml}$.),

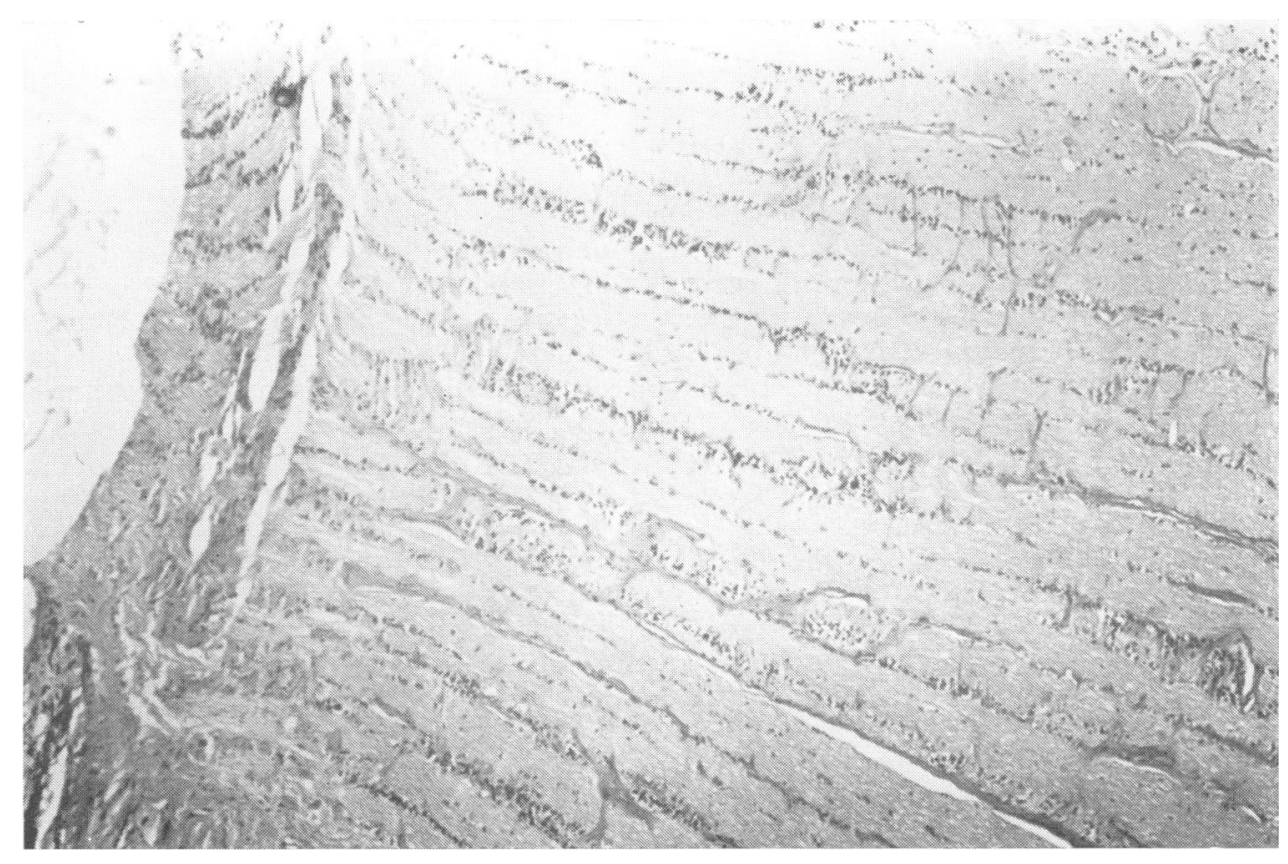

FIG. 1. Tumour invasion of the optic nerve. Optic disc and retina are on the left. Clusters of malignant cells are seen infiltrating the optic nerve, primarily between nerve fibre bundles. 


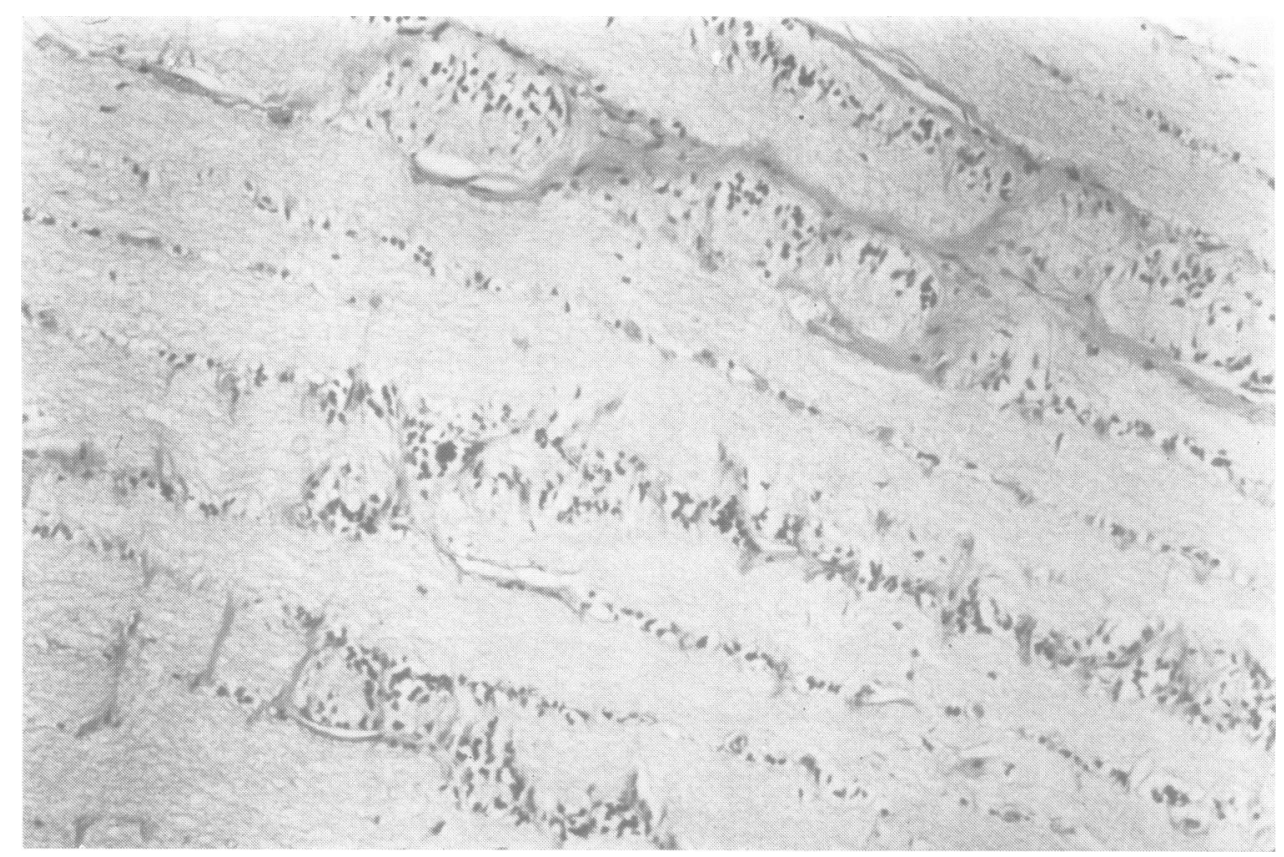

FIG. 2. Higher magnification shows small dark endometrioid sarcoma cells infiltrating the optic nerve.

and a millipore filter positive for malignant cells, confirming the diagnosis of meningeal malignancy.

She was then admitted to Stanford University Hospital where the following studies were normal: Complete blood count, erythrocyte sedimentation rate, urinalysis, blood platelet count, chemistry screening panel (glucose, phosphorus, calcium, blood urea nitrogen, uric acid, cholesterol, total protein, albumin, total bilirubin, alkaline phosphatase, lactic dehydrogenase, SGOT), antinuclear, and anticytoplasmic antibodies, radiograph of the chest, bone marrow smear, intravenous pyelogram, and barium enema. Between 9 and 30 December 1968, she was treated with $3,000 r$ of radiation to the whole brain and was given four $20 \mathrm{mg}$ injections of methotrexate intrathecally plus daily intramuscular citrovorum factor. Her spinal fluid steadily improved so that on 30 December her spinal fluid sugar was normal and no malignant cells were found on millipore examination.

Her vision, however, continued to deteriorate. Her radicular thigh pain worsened and she developed two destructive rib lesions. She was discharged from the hospital in early January almost completely blind. Cerebellar ataxia then appeared. She remained mentally alert but became increasingly depressed about the blindness and her prognosis. She died at home on 28 February 1969, of presumed intentional drug overdosage.

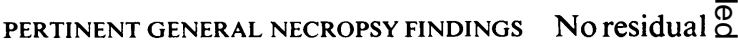
tumour was found in the irradiated region of the $\overrightarrow{\overrightarrow{0}}$ lower abdomen and pelvis but her highly anaplastic 3 endometrioid sarcoma (Jensen, Dockerty, Symmonds, and Wilson, 1966) was found to have spreado to the small bowel mesentery and to the right sixth and seventh ribs.

PERTINENT NEUROPATHOLOGICAL FINDINGS The cere- $\frac{3}{3}$. bral hemispheres, cerebellum, spinal cord, and $\delta$ meninges had a normal gross appearance. Microscopic examination showed diffuse meningeal 0 sarcomatosis of the cerebellum, spinal cord, and cauda equina, without parenchymal infiltration. No음 meningeal tumour was present over the irradiated cerebral hemispheres, which were microscopically or normal. The retinae showed no evidence of meta- $N$ static disease; the retinal arteries were normal. N Multiple sections of the optic nerves were studied ${ }_{\sigma}^{\omega}$ from the retinae to the chiasm; no meningeal tumour-cuffing of the optic nerves was present but $\stackrel{0}{?}$ there was localized infiltration by tumour of the 
optic nerves only in the intra-orbital portion, extending $6 \mathrm{~mm}$ posterior to each retin ae (Figs. 1 and 2), accompanied by mild localized axonal degeneration and loss of myelin. No tumour infiltration was found elsewhere in the optic nerves.

\section{DISCUSSION}

Blindness is the only one of many possible presenting symptoms of meningeal carcinomatosis in which this diagnosis should be considered immediately, with or without other ocular or neurological symptoms, and whether or not there is a past history of malignancy. The blindness in meningeal carcinomatosis often begins unilaterally, but usually becomes bilateral in a few days. Blindness limited to one eye has been reported only once (Walshe, 1923). Visual loss is often the first and most prominent symptom, usually progressively worsening over the course of weeks, but total blindness may occur within 48 hours (Fischer-Williams et al., 1955; Heathfield and Williams, 1956; Hughes et al., 1963; Dinsdale and Taghavy, 1964).

In the evaluation of adults with acute or subacute visual loss, when intraocular causes and temporal arteritis have been ruled out, the differential diagnosis includes bilateral infarction of the occipital lobes, compressive chiasmatic lesions, pituitary apoplexy, basilar meningitis, bilateral optic neuritis, hereditary optic atrophy, methanol intoxication, and hysteria. Most of these causes are rare and readily proved or disproved.

The finding of malignant cells on millipore examination of the spinal fluid is almost diagnostic of meningeal carcinomatosis, especially when a low level of sugar in the spinal fluid is also present (Berg, 1953; Grain and Karr, 1955; Kim and Resnick, 1965). Both the spinal fluid sugar (Kim and Resnick, 1965), and the millipore examination (Hughes et al., 1963; Dinsdale and Taghavy, 1964), however, may be normal in confirmed cases of meningeal carcinomatosis.

The prognosis in carcinomatosis of the meninges, with or without blindness, is uniformly poor, with death in weeks to a few months (Fischer-Williams et al., 1955; Grain and Karr, 1955; Dinsdale and Taghavy, 1964). $X$-ray therapy, or intrathecal methotrexate
(McKelvey, 1968) have been reported to give temporary improvement. A combination of these therapeutic methods in the present case lessened the spinal fluid abnormalities but caused no clinical improvement. No other effective treatment is available at present.

Several theories have been advanced to explain the visual loss in meningeal carcinomatosis, the leading theory being meningeal tumourcuffing in the subarachnoid space around the optic nerves. This is often prominent (FischerWilliams et al., 1955; Heathfield and Williams, 1956; Hawkins and Brown, 1963; Hughes et al. 1963; Kim and Resnick, 1965). However, similar degrees of cuffing have been reported without any visual symptoms (Hughes et al., 1963), and, in a number of cases with blindness, only minimal meningeal tumour infiltration has been found adjacent to the optic nerves (FischerWilliams et al., 1955). Chronic papilloedema from increased intracranial pressure may play a causative or contributory role in the visual loss in some patients, although the optic fundi are usually normal in cases of blindness with meningeal carcinomatosis (Fischer-Williams et al., 1955; Heathfield and Williams, 1956). Pathological changes in the occipital areas, if present, have not been of sufficient magnitude to explain the blindness in such patients and there is no evidence that blindness is one of the remote complications of cancer.

The pathophysiological causes of this type of blindness, therefore, are not yet clear. Meningeal tumour-cuffing and papilloedema, either acting alone or in combination account for many cases but some remain unexplained by either of these mechanisms. Direct neoplastic infiltration of the optic nerves has only rarely been reported. However, with only two exceptions (Terry and Dunphy, 1933; Danis and Brihaye-van Geertruyden, 1952), the intra-orbital portions of the optic nerves have not been examined in such cases of amaurosis with meningeal carcinomatosis. In the case of Terry and Dunphy (1933) blindness was the first neurological symptom in a patient with carcinoma of the lung. Heavy meningeal tumour-cuffing around the optic nerves was found from the retina to the chiasm, with neoplastic invasion of the chiasm and nearby optic nerves, not extending into the orbit. In the case of Danis and Brihaye-van Geer- 
truyden (1952), both tumour cuffing and direct neoplastic infiltration of the optic nerve were found extending from the retina to the chiasm. In the present case, no infiltration of the optic nerves and no meningeal tumour deposits were found posterior to the orbit; however, examination of the optic nerves within the orbit showed localized tumour infiltration only within $6 \mathrm{~mm}$ of each retina. The entire optic nerves, including the intraorbital portion, had been included in the radiation fields. This case thus illustrates the neuropathological importance, in patients with blindness and meningeal carcinomatosis, of examining the total extent of the optic nerves, including the intra-orbital portion adjacent to the retina.

Dr. William F. Hoyt provided valuable suggestions in the management of the case reported in this paper. The Neuropathology Department of Stanford University School of Medicine helped analyse the neuropathological material.

\section{REFERENCES}

Berg, L. (1953). Hypoglycorrhachia of non-infectious origin: diffuse meningeal neoplasia. Neurology (Minneapolis), 3, 811-824.

Danis, P., and Brihaye-van Geertruyden, M. (1952). Névrite optique rétrobulbaire bilatérale par métastases cancéreuses dans les gaines arachnoïdiennes. Acta Neurologica et Psychiatrica Belgica, 52, 345-359.
Dinsdale, H. B., and Taghavy, A. (1964). Carcinomatosis of the meninges. Canadian Medical Association Journal, 90 ,

Dixon, G. J., Kerr, A. S., and Sharp, M. E. (1946).Meningitis carcinomatosa. Report on two cases. Brain, 69, 223 232.

Fischer-Williams, M., Bosanquet, F. D., and Daniel, P. M. (1955). Carcinomatosis of the meninges. A report of three cases. Brain, 78, 42-58.

Grain, G. O., and Karr, J. P. (1955). Diffuse leptomeningeal carcinomatosis: clinical and pathologic characteristics. $\stackrel{\oplus}{+}$ Neurology (Minneapolis), 5, 706-722.

Hawkins, D., and Brown, D. (1963). Meningeal carcinoma- $\frac{\mathrm{\sigma}}{\mathrm{O}}$ tosis. Canadian Medical Association Journal, 88, 225-228.

Heathfield, K. W. G., and Williams, J. R. B. (1956). Carcinomatosis of the meninges; some clinical and pathological $\varrho$ aspects. British Medical Journal, 1, 328-330.

Hughes, I. E., Adams, J. H., and Ilbert, R. C. (1963). Invasion of the leptomeninges by tumour: the differential $\vec{\circ}$ diagnosis from tuberculous meningitis. Journal of Neurol- $\vec{\overrightarrow{ }}$ ogy, Neurosurgery, and Psychiatry, 26, 83-89.

Jensen, P. A., Dockerty, M. B., Symmonds, R. E., and Wilson, R. B. (1966). Endometrioid sarcoma ('stromal endometriosis'). American Journal of Obstetrics and Gynecology, 95, 79-90.

Katz, J. L., Valsamis, M. O., and Jampel, R. S. (1961). Ocular signs in diffuse carcinomatous meningitis. American Journal of Ophthalmology, 52, 681-690.

Kim, Y. S., and Resnick, J. S. (1965). Hypoglycorrhachia with meningeal carcinomatosis. Report of two cases. Annals of Internal Medicine, 63, 115-121.

McKelvey, E. M. (1968). Meningeal involvement with metastatic carcinoma of the breast treated with intrathec methotrexate. Cancer, 22, 576-580.

Terry, T. L., and Dunphy, E. B. (1933). Metastatic carcinori in both optic nerves simulating retrobulbar neuritiș $\omega$ Archives of Ophthalmology, 10, 611-614.

Walshe, F. M. R. (1923). Meningitis carcinomatosa. British Journal of Ophthalmology, 7, 113-123. 